\title{
A PROBLEM OF BERRY AND KNOTTED ZEROS IN THE EIGENFUNCTIONS OF THE HARMONIC OSCILLATOR
}

\author{
ALBERTO ENCISO, DAVID HARTLEY, AND DANIEL PERALTA-SALAS
}

\begin{abstract}
We prove that, given any finite link $L$ in $\mathbb{R}^{3}$, there is a highenergy complex-valued eigenfunction of the harmonic oscillator such that its nodal set contains a union of connected components diffeomorphic to $L$. This solves a problem of Berry on the existence of knotted zeros in bound states of a quantum system.
\end{abstract}

\section{INTRODUCTION}

In 2, Berry conjectures that there should be complex-valued eigenfunctions of the harmonic oscillator in $\mathbb{R}^{3}$ whose nodal set $\psi^{-1}(0)$ has knotted connected components, and raises the question of whether there can be eigenfunctions of a quantum system whose nodal set has components with higher order linking, as in the case of the Borromean rings, see Fig. 1. Furthermore, Berry remarks that it should be possible to construct these sets so that they are structurally stable in the sense that any small enough perturbation of the corresponding eigenfunction (in the $C^{k}$ norm with $k \geqslant 1$ ) still has connected components in the nodal set that are diffeomorphic to the knot or link under consideration.

As a side remark, let us recall [2, 10, that a physical motivation to study the nodal set of a quantum system is that it is the locus of destructive interference of the wave function. It is related to the existence of singularities (often called dislocations) of the phase $\operatorname{Im}(\log \psi)$ and of vortices in the current field $\operatorname{Im}(\bar{\psi} \nabla \psi)$. The existence of knotted structures of this type, especially in optics and in fluid mechanics, has recently attracted considerable attention, both from the theoretical [4, 6] and experimental [3, 9] viewpoints.

The main result of this paper solves these problems of Berry by showing that any finite link can be realized as a collection of connected components of the nodal set of a high-energy eigenfunction of the harmonic oscillator, and that the link is structurally stable in the same sense as above. Specifically, we have the following

Theorem 1.1. Let $L$ be any finite link in $\mathbb{R}^{3}$. Then one can deform it with a diffeomorphism $\Phi$ of $\mathbb{R}^{3}$ so that $\Phi(L)$ is the union of connected components of the nodal set $\psi^{-1}(0)$, where $\psi$ is a complex-valued eigenfunction of the harmonic oscillator in $\mathbb{R}^{3}$. Furthermore, the link $\Phi(L)$ is structurally stable for the function $\psi$.

We recall that the eigenfunctions of the harmonic oscillator are the squareintegrable functions $\psi$ satisfying the equation

$$
-\Delta \psi+|x|^{2} \psi=\lambda \psi
$$



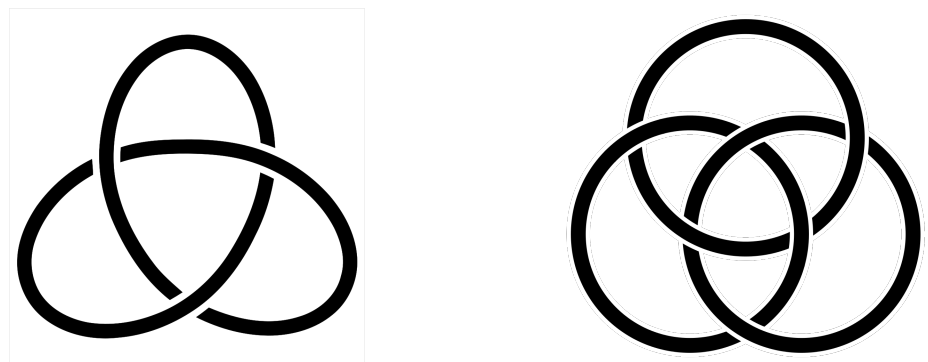

Figure 1. The problem involves showing that there are highenergy eigenfunctions $\psi$ of the harmonic oscillator realizing links, e.g. the trefoil knot and the Borromean rings depicted above, in their nodal set $\psi^{-1}(0)$.

in $\mathbb{R}^{3}$. It is well-known that the eigenvalues are of the form

$$
\lambda=2 N+3
$$

with $N$ a nonnegative integer, and that the degeneracy of the corresponding eigenspace is $\frac{1}{2}(N+1)(N+2)$.

The key idea of the proof of Theorem 1.1 is that, using techniques introduced in [4, 5, 6], one can prove that there are complex-valued solutions to the Helmholtz equation

$$
\Delta \varphi+\varphi=0
$$

in $\mathbb{R}^{3}$, such that the link $L$ is a union of connected components of the nodal set $\varphi^{-1}(0)$, up to a diffeomorphism. This is pertinent to the study of the eigenvalues of the harmonic oscillator because, in balls of radius $\lambda^{-1 / 2}$, the high-energy asymptotics of the eigenfunctions are determined by the Helmholtz equation (see e.g. [7, 8]). Heuristically, one can understand why this is true by introducing the rescaled variable $\tilde{x}:=\lambda^{1 / 2} x$, in terms of which Eq. 1.1) is read as

$$
\Delta_{\tilde{x}} \psi+\psi=\frac{|\tilde{x}|^{2} \psi}{\lambda^{2}}
$$

The way to make this precise is by computing the high-order asymptotics of the Laguerre polynomials, which govern the radial part of the eigenfunctions of the harmonic oscillator. Going over the fine details we will see that the accidental degeneracy of the eigenvalues of the harmonic oscillator is an essential ingredient of the proof too, essentially because it ensures the existence of families of isoenergetic eigenfunctions with a rich behavior in the angular variables.

The proof of Theorem 1.1 is given in Section 2, although the proofs of two technical lemmas are relegated to Sections 3 and 4 . To conclude this paper, in Section 5 we will state and discuss a higher-dimensional counterpart of the main theorem that can be proved using the same argument. 


\section{Proof of Theorem 1.1}

Let us begin by fixing an orthogonal basis of eigenfunctions associated with the harmonic oscillator Hamiltonian. Specifically, we will take

$$
\psi_{k l m}:=e^{-\frac{r^{2}}{2}} r^{l} L_{k}^{l+\frac{1}{2}}\left(r^{2}\right) Y_{l m}(\theta, \phi),
$$

where $(r, \theta, \phi)$ are spherical coordinates and we are using the standard notation for the Laguerre polynomials and the spherical harmonics. Here the indices of the eigenfunctions range over the set

$$
k \geqslant 0, \quad l \geqslant 0, \quad-l \leqslant m \leqslant l
$$

and the eigenvalue corresponding to $\psi_{k l m}$ is

$$
\lambda_{k l}:=4 k+2 l+3 .
$$

Notice that the eigenvalue is independent of $m$.

In the following lemma we will describe the behavior of the eigenfunction $\psi_{k l m}$ and its gradient for large values of $k$. To state this result, we will use the notation $e_{r}:=x / r$ for the unit vector in the radial direction and denote by $\nabla_{\mathbb{S}^{2}} Y(\theta, \phi)$ the gradient (in the unit sphere) of a function $Y(\theta, \phi)$ of the angular variables. The proof of the lemma is given in Section 3 .

Lemma 2.1. Let us fix some integers $l$ and $m$ as above. Uniformly for $r \leqslant R$, the eigenfunction $\psi_{k l m}$ admits the asymptotic expansion

$$
\begin{aligned}
\psi_{k l m}(x) & =A_{k l}\left[j_{l}\left(\sqrt{\lambda_{k l}} r\right)+O\left(\frac{1}{k}\right)\right] Y_{l m}(\theta, \phi), \\
\nabla \psi_{k l m}(x) & =\sqrt{\lambda_{k l}} A_{k l}\left[j_{l}^{\prime}\left(\sqrt{\lambda_{k l}} r\right)+O\left(\frac{1}{k}\right)\right] Y_{l m}(\theta, \phi) e_{r} \\
& +A_{k l}\left[j_{l}\left(\sqrt{\lambda_{k l}} r\right)+O\left(\frac{1}{k}\right)\right] \frac{\nabla_{\mathbb{S}^{2}} Y_{l m}(\theta, \phi)}{r},
\end{aligned}
$$

as $k \rightarrow \infty$. Here $j_{l}$ is the spherical Bessel function of order $l$ and $A_{k l}$ is a nonzero constant.

In the following lemma we construct an even complex-valued solution of the Helmholtz equation:

$$
\Delta \varphi+\varphi=0
$$

in $\mathbb{R}^{3}$ such that the link $L$ is a union of connected components of its nodal set up to a diffeomorphism. The function $\varphi$ is smooth and is conveniently given by a finite sum of spherical Bessel functions and spherical harmonics. We observe that $\varphi$ is not square-integrable but it has an optimal decay rate at infinity among all solutions to the Helmholtz equation. The proof of this lemma is presented in Section 4 and exploits ideas introduced in [4, 5, 6]:

Lemma 2.2. There are finitely many complex numbers $c_{l m}$, with $0 \leqslant l \leqslant l_{0}$ and $-l \leqslant m \leqslant l$, such that the complex-valued function

$$
\varphi:=\sum_{l=0}^{l_{0}} \sum_{m=-l}^{l} c_{l m} j_{l}(r) Y_{l m}(\theta, \phi)
$$

has the following properties:

(i) The function $\varphi$ is even, so $c_{l m}=0$ for all odd $l$. 
(ii) There is a diffeomorphism $\Phi_{1}$ of $\mathbb{R}^{3}$ such that $\Phi_{1}(L)$ is a union of connected components of the zero set $\varphi^{-1}(0)$.

(iii) $\Phi_{1}(L)$ is structurally stable. More precisely, let $S$ be a compact set containing $\Phi_{1}(L)$. Then there is some $\delta>0$ such that for any function $\varphi^{\prime}$ with $\left\|\varphi-\varphi^{\prime}\right\|_{C^{1}(S)}<\delta$ one can find a diffeomorphism $\Phi_{2}$ of $\mathbb{R}^{3}$ such that $\Phi_{2} \circ \Phi_{1}(L)$ is a collection of connected components of $\varphi^{\prime-1}(0)$ that are contained in $S$.

Let us take a large integer $\widehat{k}$ that will be fixed later, and which we assume to be larger than $\frac{l_{0}}{2}$. For each even integer $l$ smaller than $2 \widehat{k}$ we set

$$
\widehat{k}_{l}:=\widehat{k}-\frac{l}{2}
$$

so that the eigenvalue

$$
\lambda:=\lambda_{\widehat{k}_{l} l}=4 \widehat{k}+3
$$

does not depend on the choice of $l$. The desired eigenfunction $\psi$ of the harmonic oscillator can then be derived from the function $\varphi$ constructed in Lemma 2.2 by setting

$$
\psi:=\sum_{l=0}^{l_{0}} \sum_{m=-l}^{l} \frac{c_{l m}}{A_{\widehat{k}_{l} l}} \psi_{\widehat{k}_{l} l m}
$$

for a large enough number $\widehat{k}$. Notice that, by construction, $\psi$ is a smooth complexvalued function in $L^{2}\left(\mathbb{R}^{3}\right)$ that satisfies the Eq. 11.1 with $\lambda$ as in $(2.3)$. Here we have used that $c_{l m}=0$ for odd $l$, since the number $k_{l}$ defined in 2.2 is an integer only for even $l$.

Let us fix some $R>0$ such that the ball centered at the origin and of radius $R$, which we will denote by $B$, contains the link $\Phi_{1}(L)$. We claim that for any $\delta>0$ one can choose $\widehat{k}$ large enough so that

$$
\left\|\psi\left(\frac{\cdot}{\sqrt{\lambda}}\right)-\varphi(\cdot)\right\|_{C^{1}(B)}<\delta .
$$

This is a rather straightforward consequence of Lemma 2.1. Indeed, using this lemma, an elementary computation shows that

$$
\begin{aligned}
\psi_{k l m}\left(\frac{x}{\sqrt{\lambda_{k l}}}\right) & =A_{k l}\left[j_{l}(r) Y_{l m}(\theta, \phi)+O\left(\frac{1}{k}\right)\right], \\
\nabla_{x} \psi_{k l m}\left(\frac{x}{\sqrt{\lambda_{k l}}}\right) & =A_{k l}\left[j_{l}^{\prime}(r) Y_{l m}(\theta, \phi) e_{r}+\frac{j_{l}(r)}{r} \nabla_{\mathbb{S}^{2}} Y_{l m}(\theta, \phi)+O\left(\frac{1}{k}\right)\right] .
\end{aligned}
$$

Hence, substituting these asymptotic expressions in the sum for $\psi$ we find

$$
\begin{aligned}
\left|\psi\left(\frac{x}{\sqrt{\lambda}}\right)-\varphi(x)\right| & \leqslant \sum_{l=0}^{l_{0}} \sum_{m=-l}^{l} c_{l m}\left|\frac{1}{A_{\widehat{k}_{l} l}} \psi_{\widehat{k}_{l} l m}\left(\frac{x}{\sqrt{\lambda}}\right)-j_{l}(r) Y_{l m}(\theta, \phi)\right| \\
& =\sum_{l=0}^{l_{0}} \sum_{m=-l}^{l} c_{l m} O\left(\frac{1}{\widehat{k}_{l}}\right) \leqslant \frac{C}{\widehat{k}-\frac{l_{0}}{2}} \leqslant \frac{C}{\widehat{k}}
\end{aligned}
$$


provided $\widehat{k}$ is much larger than $\frac{l_{0}}{2}$ and $|x|<R$. An analogous argument shows

$$
\left|\nabla_{x} \psi\left(\frac{x}{\sqrt{\lambda}}\right)-\nabla_{x} \varphi(x)\right| \leqslant \frac{C}{\widehat{k}}
$$

so the estimate (2.4) follows provided $\widehat{k}$ is large enough.

Item (iii) in Lemma 2.2 ensures that, if $\delta$ is small enough, the function $\psi(\cdot / \sqrt{\lambda})$ has a collection of connected components in its nodal set $\{\psi(\cdot / \sqrt{\lambda})=0\}$ given by the link $\Phi_{2} \circ \Phi_{1}(L)$, where $\Phi_{2}$ is a diffeomorphism of $\mathbb{R}^{3}$ and $\Phi_{2} \circ \Phi_{1}(L)$ is contained in $B$. Item (iii) also ensures that the link $\Phi_{2} \circ \Phi_{1}(L)$ is structurally stable for the eigenfunction $\psi$. This implies that the rescaled link $\Phi_{3} \circ \Phi_{2} \circ \Phi_{1}(L)$ is a union of structurally stable connected components of $\psi^{-1}(0)$, where $\Phi_{3}$ denotes the diffeomorphism of $\mathbb{R}^{3}$ given by the rescaling

$$
\Phi_{3}(x):=\frac{x}{\sqrt{\lambda}} .
$$

The theorem then follows by setting $\Phi:=\Phi_{3} \circ \Phi_{2} \circ \Phi_{1}$.

Remark 2.3. It is worth noting that the fact that the function $\varphi$ is even was key to constructing the radial quantum number $k_{l}$ via Eq. 2.2. A straightforward modification of the argument enables us to consider the case where $\varphi$ is odd. As is well known, all eigenfunctions of the harmonic oscillator must have a definite parity. In particular the nodal set of the eigenfunction $\psi$ contains (at least) two copies of the link $\Phi(L)$ as the link $\Phi_{1}(L)$ constructed in the proof of Lemma 2.2 is contained in the positive octant of $\mathbb{R}^{3}$, which implies that so is $\Phi(L)$. Moreover, $\Phi(L)$ is contained in a small ball of radius $R \lambda^{-1 / 2}$.

\section{Proof of Lemma 2.1}

The lemma essentially follows from Hilb's asymptotic formula for the Laguerre polynomials [12, Theorem 8.22.4]:

$$
\begin{gathered}
e^{-\frac{r^{2}}{2}} r^{l} L_{k}^{l+\frac{1}{2}}\left(r^{2}\right)=A_{k l} j_{l}\left(\sqrt{\lambda_{k l}} r\right)+O\left(k^{\frac{l-1}{2}}\right), \\
\frac{d}{d r}\left[e^{-\frac{r^{2}}{2}} r^{l} L_{k}^{l+\frac{1}{2}}\left(r^{2}\right)\right]=\sqrt{\lambda_{k l}}\left[A_{k l} j_{l}^{\prime}\left(\sqrt{\lambda_{k l}} r\right)+O\left(k^{\frac{l-1}{2}}\right)\right],
\end{gathered}
$$

with

$$
A_{k l}:=\frac{2}{\sqrt{\pi}}\left(\frac{\sqrt{\lambda_{k l}}}{2}\right)^{-l} \frac{\Gamma\left(k+l+\frac{3}{2}\right)}{k !} .
$$

This formula holds uniformly for $r \leqslant R$. (In fact, the formula for the derivative does not appear in the above reference, but it is standard - and easy to provethat this asymptotic formula can be derived term by term).

The asymptotic expansion for $\psi_{k l m}$ written in the lemma follows from the identity 2.1 and the fact that the constant $A_{k l}$ can be estimated for large $k$ as

$$
A_{k l}=\frac{2}{\sqrt{\pi}} k^{\frac{l+1}{2}}+O\left(k^{\frac{l-1}{2}}\right) .
$$

This is an elementary computation using Stirling's formula for the factorial and the identity

$$
\Gamma\left(k+l+\frac{3}{2}\right)=\frac{\sqrt{\pi}(2 k+2 l+2) !}{2^{2 k+2 l+2}(k+l+1) !} .
$$




\section{Proof of Lemma 2.2}

Let $B$ be a ball centered at the origin that contains the link $L$. There is no loss of generality in assuming that $L$ is contained in the positive octant of $\mathbb{R}^{3}$, that is,

$$
L \subset B \cap\left\{x_{1}>0, x_{2}>0, x_{3}>0\right\} .
$$

An easy application of Whitney's approximation theorem ensures that, by perturbing the link a little if necessary, we can assume that it is a real analytic submanifold of $\mathbb{R}^{3}$.

Let us denote by $L_{\alpha}$ the connected components of $L$, with the index $\alpha$ taking values in a finite set $A$. Each component $L_{\alpha}$ is an analytic closed curve without selfintersections. Our next goal is to write the curve $L_{\alpha}$ as the transverse intersection of two surfaces $\Sigma_{\alpha}^{1}$ and $\Sigma_{\alpha}^{2}$.

Since any closed curve in $\mathbb{R}^{3}$ has trivial normal bundle [11, there exists an analytic submersion $\Theta_{\alpha}: W_{\alpha} \rightarrow \mathbb{R}^{2}$, where $W_{\alpha}$ is a tubular neighborhood of $L_{\alpha}$ and $\Theta_{\alpha}^{-1}(0)=L_{\alpha}$. We can then take the analytic surfaces $\Sigma_{\alpha}^{1}:=\Theta_{\alpha}^{-1}((-1,1) \times\{0\}) \subset$ $W_{\alpha}$ and $\Sigma_{\alpha}^{2}:=\Theta_{\alpha}^{-1}(\{0\} \times(-1,1)) \subset W_{\alpha}$. Since $\Theta_{\alpha}$ is a submersion, these surfaces intersect transversally at $L_{\alpha}=\Sigma_{\alpha}^{1} \cap \Sigma_{\alpha}^{2}$.

Now that we have expressed the component $L_{\alpha}$ as the intersection of two real analytic surfaces $\Sigma_{a}^{1}$ and $\Sigma_{\alpha}^{2}$, we can consider the following Cauchy problems, with $j=1,2$ :

$$
\Delta u_{\alpha}^{j}+u_{\alpha}^{j}=0,\left.\quad u_{\alpha}^{j}\right|_{\Sigma_{\alpha}^{j}}=0,\left.\quad \partial_{\nu} u_{\alpha}^{j}\right|_{\Sigma_{\alpha}^{j}}=1 .
$$

Here $\partial_{\nu}$ denotes a normal derivative at the corresponding surface. The CauchyKowalewski theorem then grants the existence of solutions $u_{\alpha}^{j}$ to this Cauchy problem in the closure of small neighborhoods $U_{\alpha}^{j}$ of each surface $\Sigma_{\alpha}^{j}$. We can safely assume that the tubular neighborhoods $U_{\alpha}^{1} \cap U_{\alpha}^{2}$ are small enough so that the neighborhoods corresponding to distinct components are disjoint. Now we take the union of these pairwise disjoint tubular neighborhoods,

$$
U:=\bigcup_{\alpha \in A}\left(U_{\alpha}^{1} \cap U_{\alpha}^{2}\right)
$$

and define a complex-valued function $\hat{\varphi}$ on the set $U$ as

$$
\left.\hat{\varphi}\right|_{U_{\alpha}^{1} \cap U_{\alpha}^{2}}:=u_{\alpha}^{1}+i u_{\alpha}^{2} .
$$

The following properties of $\hat{\varphi}$ are clear from the construction:

(i) $\hat{\varphi}$ satisfies the equation

$$
\Delta \hat{\varphi}+\hat{\varphi}=0
$$

in the tubular neighborhood $U$ of the link $L$. We can assume without loss of generality that $U$ is contained in the positive octant $B \cap\left\{x_{1}>0, x_{2}>\right.$ $\left.0, x_{3}>0\right\}$, as is the link $L$.

(ii) $U$ can be taken small enough so that the nodal set of $\hat{\varphi}$ is precisely $L$, i.e., $L=\hat{\varphi}^{-1}(0)$.

(iii) The intersection of the zero sets of the real and imaginary parts of $\hat{\varphi}$ on $L$ is transverse, i.e.,

$$
\operatorname{rank}(\nabla \operatorname{Re} \hat{\varphi}(x), \nabla \operatorname{Im} \hat{\varphi}(x))=2
$$

for all $x \in L$. 
Let us agree to say that a subset of $\mathbb{R}^{3}$ is symmetric if it is invariant under the inversion $x \mapsto-x$, and denote by $-U$ the image of the set $U$ under this map. Since $U$ is contained in the positive octant, $U \cap-U=\emptyset$.

Let us then define an even function $\varphi^{\prime}$ in the symmetric set

$$
U^{\prime}:=U \cup-U,
$$

as

$$
\varphi^{\prime}(x):= \begin{cases}\hat{\varphi}(x) & \text { if } x \in U, \\ \hat{\varphi}(-x) & \text { if } x \in-U .\end{cases}
$$

By construction, $\varphi^{\prime}$ satisfies the Helmholtz equation

$$
\Delta \varphi^{\prime}+\varphi^{\prime}=0
$$

in $U^{\prime}$ and its nodal set consists of $L$ and its mirror image under the inversion $x \mapsto-x$.

Denote by $S$ a symmetric closed subset of $U^{\prime}$ whose interior contains the link $L$. Our next goal is to construct a solution of the Helmholtz equation in $\mathbb{R}^{3}$ that approximates the local solution $\varphi^{\prime}$ in the set $S$. To this end, let us take a smooth even function $\chi: \mathbb{R}^{3} \rightarrow \mathbb{R}$ equal to 1 in a neighborhood of $S$ and identically zero outside $U^{\prime}$, and define a smooth extension $\varphi_{0}$ of the function $\varphi^{\prime}$ to $\mathbb{R}^{3}$ by setting $\varphi_{0}:=\chi \varphi^{\prime}$, which is an even function too. Denote by

$$
G(x):=\frac{\cos |x|}{4 \pi|x|}
$$

the Green's function of the operator $\Delta+1$ in $\mathbb{R}^{3}$, which satisfies the distributional equation

$$
\Delta G+G=-\delta_{0}
$$

with $\delta_{0}$ the Dirac measure supported at 0 . Since $\varphi_{0}$ is compactly supported, we obviously have

$$
\varphi_{0}(x)=\int_{\mathbb{R}^{3}} G\left(x-x^{\prime}\right) \rho\left(x^{\prime}\right) d x^{\prime}
$$

with $\rho:=-\Delta \varphi_{0}-\varphi_{0}$. The complex-valued function $\rho$ is even and its support is contained in the set $U^{\prime} \backslash S$. Therefore, an easy continuity argument ensures that one can approximate the integral (4.3) uniformly in the compact set $S$ by a finite Riemann sum of the form

$$
\varphi_{1}(x):=\sum_{j=-J}^{J} \rho_{j} G\left(x-x_{j}\right) .
$$

Specifically, for any $\delta>0$ there is a large integer $J$, complex numbers $\rho_{j}$ and points $x_{j} \in U^{\prime} \backslash S$ such that the finite sum 4.4 satisfies

$$
\left\|\varphi_{1}-\varphi^{\prime}\right\|_{C^{0}(S)}<\delta \text {. }
$$

By the symmetry of the integrand, these quantities can be chosen such that $\rho_{0}=0$, $\rho_{-j}=\rho_{j}$ and $x_{-j}=-x_{j}$ for $j>0$, thus guaranteeing that $\varphi_{1}$ is an even function. Here we have used that $\varphi_{0}=\varphi^{\prime}$ in $S$.

In the following lemma we show how to "sweep" the singularities of the function $\varphi_{1}$ in order to approximate it in the set $S$ by another function $\varphi_{2}$ whose singularities are contained in the complement of the ball $B$. The proof is based on a duality argument and the Hahn-Banach theorem. 
Lemma 4.1. For any $\delta>0$, there is a finite set of points $\left\{z_{j}\right\}_{j=-J^{\prime}}^{J^{\prime}}$ in $\mathbb{R}^{3} \backslash \bar{B}$ and complex numbers $c_{j}$ such that the finite linear combination

$$
\varphi_{2}(x):=\sum_{j=-J^{\prime}}^{J^{\prime}} c_{j} G\left(x-z_{j}\right)
$$

approximates the function $\varphi_{1}$ uniformly in $S$ :

$$
\left\|\varphi_{2}-\varphi_{1}\right\|_{C^{0}(S)}<\delta
$$

Moreover,

$$
c_{0}=0, \quad z_{-j}=-z_{j}, \quad c_{-j}=c_{j},
$$

for all $j>0$, so that $\varphi_{2}$ is an even function.

Proof. Consider the space $\mathcal{U}$ of all complex-valued functions that are linear combinations of the form (4.6), not necessarily even, where $z_{j}$ can be any point in $\mathbb{R}^{3} \backslash \bar{B}$ and the constants $c_{j}$ take arbitrary complex values. Restricting these functions to the set $S, \mathcal{U}$ can be regarded as a subspace of the Banach space $C^{0}(S)$ of continuous complex-valued functions on $S$.

By the Riesz-Markov theorem, the dual of $C^{0}(S)$ is the space $\mathcal{M}(S)$ of the finite complex-valued Borel measures on $\mathbb{R}^{3}$ whose support is contained in the set $S$. Let us take any measure $\mu \in \mathcal{M}(S)$ such that $\int_{\mathbb{R}^{3}} f d \mu=0$ for all $f \in \mathcal{U}$. Let us now define a complex-valued function $F \in L_{\text {loc }}^{1}\left(\mathbb{R}^{3}\right)$ as

$$
F(x):=\int_{\mathbb{R}^{3}} G(\tilde{x}-x) d \mu(\tilde{x}),
$$

so that $F$ satisfies the equation

$$
\Delta F+F=-\mu .
$$

Notice that $F$ is identically zero on $\mathbb{R}^{3} \backslash \bar{B}$ by the definition of the measure $\mu$ and that $F$ satisfies the elliptic equation

$$
\Delta F+F=0
$$

in $\mathbb{R}^{3} \backslash S$, so $F$ is analytic in this set. Hence, since $\mathbb{R}^{3} \backslash S$ is connected and contains the set $\mathbb{R}^{3} \backslash B$, by analyticity the function $F$ must vanish on the complement of $S$. It then follows that the measure $\mu$ also annihilates any complex-valued function of the form $\rho_{j} G\left(x-x_{j}\right)$ because, as the points $x_{j}$ do not belong to $S$,

$$
0=\rho_{j} F\left(x_{j}\right)=\int_{\mathbb{R}^{3}} \rho_{j} G\left(x-x_{j}\right) d \mu(x) .
$$

Therefore

$$
\int_{\mathbb{R}^{3}} \varphi_{1} d \mu=0
$$

which implies that $\varphi_{1}$ can be uniformly approximated on $S$ by elements of the subspace $\mathcal{U}$, due to a consequence of the Hahn-Banach theorem. Accordingly, there is a finite set of points $\left\{z_{j}\right\}_{j=1}^{J^{\prime}}$ in $\mathbb{R}^{3} \backslash \bar{B}$ and complex numbers $c_{j}$ such that the function

$$
\hat{\varphi}_{2}(x):=\sum_{j=1}^{J^{\prime}} 2 c_{j} G\left(x-z_{j}\right)
$$


approximates the function $\varphi_{1}$ uniformly in $S$ :

$$
\left\|\hat{\varphi}_{2}-\varphi_{1}\right\|_{C^{0}(S)}<\delta .
$$

The lemma then follows by setting

$$
\varphi_{2}(x):=\sum_{j=1}^{J^{\prime}} c_{j} G\left(x-z_{j}\right)+\sum_{j=1}^{J^{\prime}} c_{j} G\left(x+z_{j}\right)=: \sum_{j=-J^{\prime}}^{J^{\prime}} c_{j} G\left(x-z_{j}\right)
$$

where $c_{0}=0, c_{-j}=c_{j}$ and $z_{-j}=z_{j}$. Indeed, since $S$ is a symmetric set, we have that, for all $x \in S$,

$\varphi_{2}(x)-\varphi_{1}(x)=\varphi_{2}(x)-\frac{\varphi_{1}(x)+\varphi_{1}(-x)}{2}=\frac{\hat{\varphi}_{2}(x)-\varphi_{1}(x)}{2}+\frac{\hat{\varphi}_{2}(-x)-\varphi_{1}(-x)}{2}$,

which implies the desired estimate

$$
\left\|\varphi_{2}-\varphi_{1}\right\|_{C^{0}(S)} \leqslant \frac{1}{2}\left\|\hat{\varphi}_{2}-\varphi_{1}\right\|_{C^{0}(S)}+\frac{1}{2}\left\|\hat{\varphi}_{2}-\varphi_{1}\right\|_{C^{0}(S)}<\delta .
$$

Notice that we have used the identity $\varphi_{1}(x)=\varphi_{1}(-x)$

To complete the proof of the lemma, notice that the even complex-valued function $\varphi_{2}$ constructed in Lemma 4.1 satisfies

$$
\Delta \varphi_{2}+\varphi_{2}=0
$$

in the ball $B$, whose interior contains $S$. Let us take spherical coordinates $(r, \theta, \varphi)$ in the ball $B$. Expanding the function $\varphi_{2}$ (with respect to the angular variables) in a series of spherical harmonics and using Eq. (4.8), we immediately obtain that $\varphi_{2}$ can be written in the ball as a Fourier-Bessel series of the form

$$
\varphi_{2}=\sum_{l=0}^{\infty} \sum_{m=-l}^{l} c_{l m} j_{l}(r) Y_{l m}(\theta, \varphi)
$$

Since $\varphi_{2}$ is even, we have that $c_{l m}=0$ for all odd $l$. As before, $j_{l}$ denotes a spherical Bessel function.

Since the above series converges in $L^{2}(B)$, for any $\delta>0$ there is an integer $l_{0}$ such that the finite sum

$$
\varphi:=\sum_{l=0}^{l_{0}} \sum_{m=-l}^{l} c_{l m} j_{l}(r) Y_{l m}(\theta, \varphi)
$$

approximates the function $\varphi_{2}$ in an $L^{2}$ sense:

$$
\left\|\varphi-\varphi_{2}\right\|_{L^{2}(B)}<\delta .
$$

By the properties of spherical Bessel functions, the complex-valued function $\varphi$ is smooth in $\mathbb{R}^{3}$ and satisfies the equation

$$
\Delta \varphi+\varphi=0
$$

in the whole space.

Given any smaller ball $B^{\prime}$, properly contained in $B$ and in turn containing the set $S$, standard elliptic estimates allow us to pass from the $L^{2}$ bound 4.9 to a uniform estimate

$$
\left\|\varphi-\varphi_{2}\right\|_{C^{0}\left(B^{\prime}\right)}<C \delta .
$$


From this inequality and the bounds 4.5 and 4.7 we infer

$$
\left\|\varphi-\varphi^{\prime}\right\|_{C^{0}(S)}<C \delta \text {. }
$$

Moreover, since $\varphi^{\prime}$ also satisfies the Helmholtz equation in a neighborhood of the compact set $S$ (cf. Eq. (4.2)), standard elliptic estimates again imply that the uniform estimate (4.11) can be promoted to the $C^{1}$ bound

$$
\left\|\varphi-\varphi^{\prime}\right\|_{C^{1}(S)}<C \delta .
$$

Finally, since the link $L$ is a union of components of the the nodal set of $\varphi^{\prime}$ and satisfies the transversality condition (4.1), the estimate (4.12) and a direct application of Thom's isotopy theorem [1, Theorem 20.2] imply that there is a diffeomorphism $\Phi_{1}$ of $\mathbb{R}^{3}$ such that $\Phi_{1}(L)$ is a union of components of the zero set $\varphi^{-1}(0)$. Moreover, the diffeomorphism $\Phi_{1}$ is $C^{1}$-close to the identity and different from the identity just in a small neighborhood of $L$, so we can safely assume that $\Phi_{1}(L)$ is contained in $B$. The structural stability of the link $\Phi_{1}(L)$ for the function $\varphi$ also follows from Thom's isotopy theorem and the fact that $\varphi$ satisfies the transversality condition

$$
\operatorname{rank}(\nabla \operatorname{Re} \varphi(x), \nabla \operatorname{Im} \varphi(x))=2
$$

for all $x \in \Phi_{1}(L)$. This last equation is a consequence of the $C^{1}$-estimate 4.12 , the fact that the function $\varphi^{\prime}$ satisfies the transversality estimate 4.1) by definition, and the fact that transversality is an open property under $C^{1}$-small perturbations. The lemma then follows.

\section{A REMARK ABOUt THE HIGHER DIMENSIONAL COUNTERPART}

Following Berry, we have considered the construction of a complex-valued eigenfunction (or two real-valued eigenfunctions) of the harmonic oscillator in three dimensions with a prescribed nodal set of codimension 2 (that is, a link). It is worth mentioning that essentially the same argument enables us to construct $n$ eigenfunctions of the harmonic oscillator in $\mathbb{R}^{d}$ with a prescribed nodal set of codimension $n$.

However, a technical condition makes the statement considerably more involved in the general case. This condition is associated with the requirement that the level set be structurally stable. In the situation covered by the main theorem, the structural stability follows from the important equation 4.1, which plays a crucial role in the proof. The higher dimensional analog of that relation would then be the requirement that

$$
\operatorname{rank}\left(\nabla \psi_{1}(x), \ldots, \nabla \psi_{n}(x)\right)=n,
$$

for all $x$ in the prescribed codimension- $n$ nodal set in $\mathbb{R}^{d}$, where $\psi_{1}, \ldots, \psi_{n}$ would be real-valued eigenfunctions of the harmonic oscillator. For this condition to hold, a topological obstruction is that the normal bundle of the set $L$ that we want to prescribe in the nodal set must be trivial. Geometrically, this is equivalent to the assertion that a small tubular neighborhood of the submanifold $L$ must be diffeomorphic to $L \times \mathbb{R}^{n}$.

Hence we are led to the following result. Since a link always has trivial normal bundle [11], the main theorem corresponds exactly to the case $d=3$ and $n=2$. 
Theorem 5.1. Let $L$ be a finite disjoint union of codimension-n compact submanifolds of $\mathbb{R}^{d}$ with trivial normal bundle, and $d \geqslant 3$. If $n=1$, we also assume that $L$ is connected. Then for any large enough eigenvalue $\lambda$ of the harmonic oscillator in $\mathbb{R}^{d}$ there are $n$ real-valued eigenfunctions $\psi_{1}, \ldots, \psi_{n}$ with eigenvalue $\lambda$ and a diffeomorphism $\Phi$ of $\mathbb{R}^{d}$ such that $\Phi(L)$ is the union of connected components of the joint nodal set $\psi_{1}^{-1}(0) \cap \cdots \cap \psi_{n}^{-1}(0)$. Furthermore, $\Phi(L)$ is structurally stable.

We note that when $d \geqslant 3$ the eigenfunctions of the harmonic oscillator are given by

$$
\psi_{k l m}=e^{-\frac{r^{2}}{2}} r^{l} L_{k}^{l+\frac{d-2}{2}}\left(r^{2}\right) Y_{l m}(\omega),
$$

and the corresponding eigenvalues are $\lambda_{k l}=4 k+2 l+d$. Here $\omega:=x / r$ is a point in $\mathbb{S}^{d-1}$ and $Y_{l m}$ are the spherical harmonics in $\mathbb{S}^{d-1}$ with frequency $l(l+d-2)$, with $l \geqslant 0, m \equiv\left(m_{1}, \ldots, m_{d-2}\right)$ and

$$
\left|m_{1}\right| \leqslant m_{2} \leqslant \cdots \leqslant m_{d-2} \leqslant l .
$$

Using again Hilb's asymptotic formula for the Laguerre polynomials 12, Theorem 8.22.4], we get the asymptotic expressions of Lemma 2.1 generalized to any $d \geqslant 3$ :

$$
\begin{aligned}
\psi_{k l m}(x) & =A_{k l}^{d}\left[j_{l}^{d}\left(\sqrt{\lambda_{k l}} r\right)+O\left(k^{-\min \left(\frac{d+1}{4}, 2\right)}\right)\right] Y_{l m}(\omega), \\
\nabla \psi_{k l m}(x) & =\sqrt{\lambda_{k l}} A_{k l}^{d}\left[\left(j_{l}^{d}\right)^{\prime}\left(\sqrt{\lambda_{k l}} r\right)+O\left(k^{-\min \left(\frac{d+1}{4}, 2\right)}\right)\right] Y_{l m}(\theta, \phi) e_{r} \\
& +A_{k l}^{d}\left[j_{l}^{d}\left(\sqrt{\lambda_{k l}} r\right)+O\left(k^{-\min \left(\frac{d+1}{4}, 2\right)}\right)\right] \frac{\nabla_{\mathbb{S}^{d}-1} Y_{l m}(\omega)}{r},
\end{aligned}
$$

where $j_{l}^{d}$ denote the hyperspherical Bessel functions, which satisfy the radial Helmholtz equation in $\mathbb{R}^{d}$, and the constants $A_{k l}^{d}$ are given by

$$
A_{k l}^{d}:=\frac{1}{\Gamma\left(\frac{d}{2}\right)}\left(\frac{\sqrt{\lambda_{k l}}}{2}\right)^{-l} \frac{\Gamma\left(k+l+\frac{d}{2}\right)}{k !} .
$$

The proof of Lemma 2.2 goes exactly as in Section 4 when the codimension is $n \geqslant 2$. In this case, we use the Cauchy-Kolaweski theorem with data on hypersurfaces $\Sigma_{\alpha}^{j}(1 \leqslant j \leqslant n)$, intersecting at a given component $L_{\alpha}$ transversally, to define functions $u_{a}^{j}$. When $n=1$, however, the construction of the real-valued function $u$ (analogous to the functions $u_{\alpha}^{j}$ considered in Section 4, where we are dropping the sub- and superscripts because $L$ is now connected and $n=1$ ) cannot be performed using the Cauchy-Kowalweski theorem because otherwise $u$ would be defined in a small neighborhood $U$ of $L$. As the complement of $U$ would not be connected, the proof of Lemma 4.1 does not carry over to this case. To circumvent this difficulty, we take $U$ to be the precompact domain bounded by $L$ and define $u$ in $s U$ as the first Dirichlet eigenfunction of the Laplacian in this domain, where $s$ is a scale factor chosen so that the first Dirichlet eigenvalue of $s U$ is 1 . The details are as in [5, Appendix A]. The rest of the proof remains essentially unchanged.

In particular, in three dimensions the general result yields not only Theorem 1.1 but also the following

Corollary 5.2. Let $L$ be a compact surface in $\mathbb{R}^{3}$. Then for any large enough eigenvalue $\lambda$ of the harmonic oscillator in $\mathbb{R}^{3}$ there is a real-valued eigenfunction $\psi$ of the harmonic oscillator with energy $\lambda$ and a diffeomorphism $\Phi$ of $\mathbb{R}^{3}$ such that $\Phi(L)$ is a connected component of the nodal set $\psi^{-1}(0)$. Furthermore, $\Phi(L)$ is structurally stable. 


\section{ACKNOWLEDGMENTS}

The authors are supported by the ERC Starting Grants 633152 (A.E.) and 335079 (D.H. and D.P.-S.). This work is supported in part by the ICMAT-Severo Ochoa grant SEV-2011-0087.

\section{REFERENCES}

1. R. Abraham, J. Robbin, Transversal Mappings and Flows, Benjamin, New York, 1967.

2. M. Berry, Knotted zeros in the quantum states of hydrogen, Found. Phys. 31 (2001) 659-667.

3. M.R. Dennis, R.P. King, B. Jack, K. O'Holleran, M.J. Padgett, Isolated optical vortex knots, Nature Phys. 6 (2010) 118-121.

4. A. Enciso, D. Peralta-Salas, Knots and links in steady solutions of the Euler equation, Ann. of Math. 175 (2012) 345-367.

5. A. Enciso, D. Peralta-Salas, Submanifolds that are level sets of solutions to a second-order elliptic PDE, Adv. Math. 249 (2013) 204-249.

6. A. Enciso, D. Peralta-Salas, Existence of knotted vortex tubes in steady Euler flows, Acta Math. 214 (2015) 61-134.

7. A. Eremenko, D. Jakobson, N. Nadirashvili, On nodal sets and nodal domains on $\mathbb{S}^{2}$ and $\mathbb{R}^{2}$, Ann. Inst. Fourier (Grenoble) 57 (2007) 2345-2360.

8. D. Jakobson, N. Nadirashvili, J. Toth, Geometric properties of eigenfunctions, Russian Math. Surveys 56 (2001) 1085-1105.

9. D. Kleckner, W.T.M. Irvine, Creation and dynamics of knotted vortices, Nature Phys. 9 (2013) 253-258.

10. L.H. Kauffman, S.J. Lomonaco, Quantum knots, Proc. SPIE 5436 (2004) 268-284.

11. W.S. Massey, On the normal bundle of a sphere imbedded in Euclidean space, Proc. Amer. Math. Soc. 10 (1959) 959-964.

12. G. Szegő, Orthogonal Polynomials, AMS, Providence, 1975.

Instituto de Ciencias Matemáticas, Consejo Superior de Investigaciones Científicas, 28049 MADRID, SPAIN

E-mail address: aenciso@icmat.es, david.hartley@icmat.es, dperalta@icmat.es 